JOURNAL OF SYNCHROTRON RADIATION
Received 30 November 2016

Accepted 2 December 2016

Keywords: high-energy-resolution analysers; inelastic X-ray scattering; bent crystals.

\section{Study on the reflectivity properties of spherically bent analyser crystals. Corrigendum}

\author{
Ari-Pekka Honkanen, ${ }^{\text {a* }}$ Roberto Verbeni, ${ }^{b}$ Laura Simonelli, ${ }^{b}$ Marco Moretti Sala, \\ Giulio Monaco $^{\mathrm{b}, \mathrm{c}}$ and Simo Huotari ${ }^{\mathrm{a}}$
}

${ }^{\mathbf{a}}$ Department of Physics, PO Box 64, Fl-00014 Helsinki, Finland, ${ }^{\mathbf{b}}$ European Synchrotron Radiation Facility, BP 220, F-38043 Grenoble Cedex, France, and ' Physics Department, University of Trento, Via Sommarive 14, 38123 Povo (TN), Italy. *Correspondence e-mail: ari-pekka.honkanen@helsinki.fi

Corrections to the paper by Honkanen et al. (2014). [J. Synchrotron Rad. 21, 104-110] are made.

After the publication of our manuscript (Honkanen et al., 2014), we have learned of the presence of certain shortcomings in the computation of the theoretical reflectivity curves:

(i) The largest aperture used in the measurements of the reflectivity curves was $100 \mathrm{~mm}$ (i.e. full analyser) instead of $86 \mathrm{~mm}$.

(ii) The approximation used for the deviation parameter in the one-dimensional Takagi-Taupin equation was not sufficiently accurate near the backscattering, affecting the shapes of the curves on the right-hand side.

(iii) The incident bandwidth of the used $(+,-,-,+)$ monochromator configuration is not modelled well enough by a Gaussian function.

While not affecting the main conclusions of our work, these errors do alter the shape of the theoretical predictions and can be improved upon. They were addressed as follows:

(i) The incorrect aperture size was changed from $86 \mathrm{~mm}$ to $100 \mathrm{~mm}$.

(ii) The depth-depended Takagi-Taupin curves were computed with the Python code presented by Honkanen et al. (2016) that uses a different formulation for the deviation parameter.

(iii) The incident bandwidth was computed by combining the single-crystal reflectivity curves of the monochromator crystals.

The corrected theoretical curves in conjunction with the measured ones are presented in Fig. 1 and the agreement of the experiment and theory is improved from the original one.

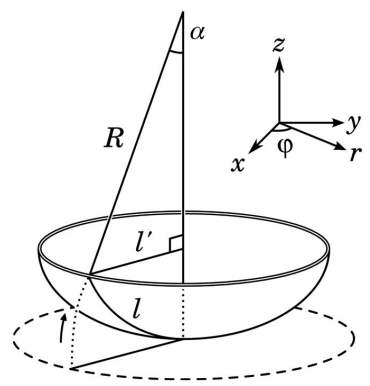

(C) 2017 International Union of Crystallography 


\section{addenda and errata}
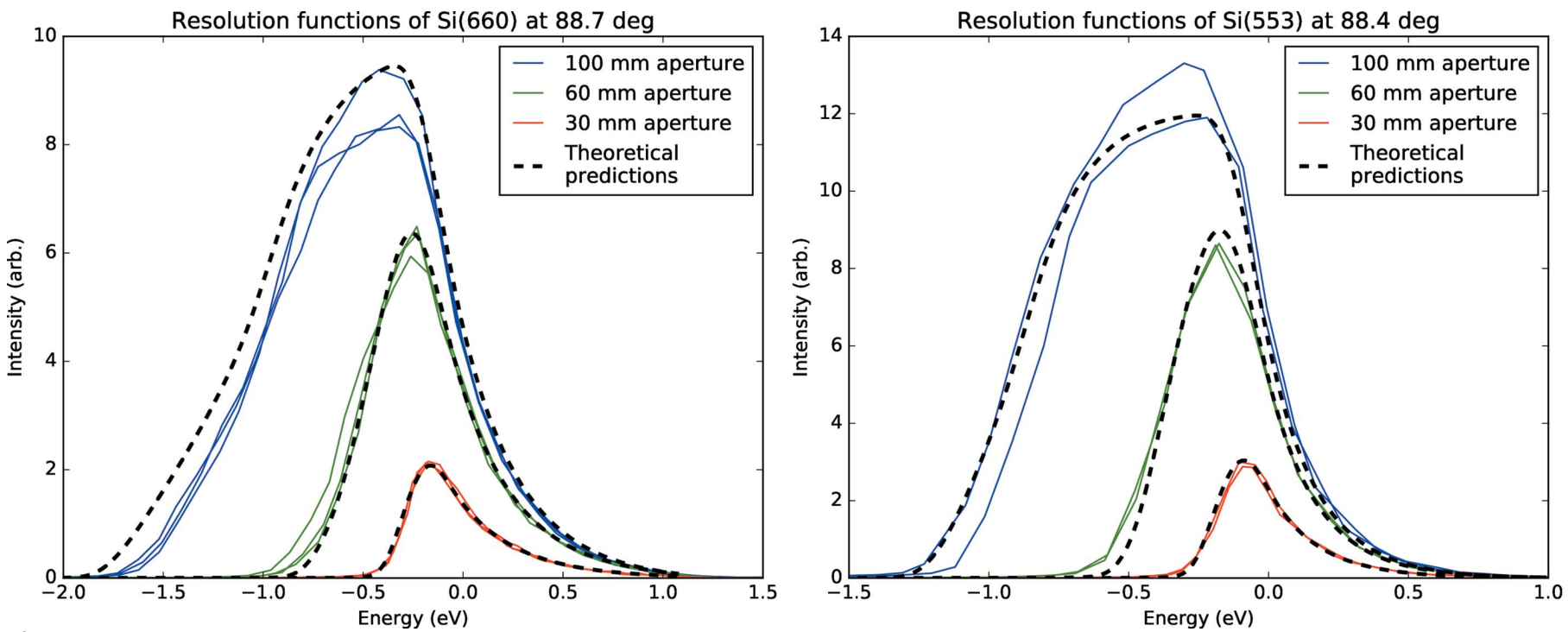

Figure 1

Corrected predictions and measured reflectivity curves of $\mathrm{Si}(660)$ and $\mathrm{Si}(553)$ analysers. Three different curves are shown for each analyser corresponding to different mask aperture sizes.

\section{References}

Honkanen, A.-P., Monaco, G. \& Huotari, S. (2016). J. Appl. Cryst. 49, 1284-1289.
Honkanen, A.-P., Verbeni, R., Simonelli, L., Moretti Sala, M., Monaco, G. \& Huotari, S. (2014). J. Synchrotron Rad. 21, 104-110. 